\title{
Thermal balance and metabolic rate during upper respiratory tract infection in infants
}

\author{
Peter J Fleming, Thelma Howell, Mark Clements, Jane Lucas
}

\begin{abstract}
Sequential recordings were made in the first five months after birth of metabolic rate, environmental temperature, and body temperature during sleep at home in 17 infants, each with an older sibling. Further recordings were made whenever an older sibling developed an upper respiratory tract infection (URTI), again four to six days later, and again two weeks later, aiming to achieve recordings before, during, and after an URTI in the infant. The temperature of the room and wrapping of the infant were determined according to their usual practice by the parents.
\end{abstract}

Parents added appropriate wrapping to achieve thermal neutrality based on our calculated values and the measured oxygen consumption.

In five of the six infants who developed an URTI in the first three months after birth there was no change or a decrease in metabolic rate at the time of the infection; for infants older than 3 months the metabolic rate increased in three of the five episodes recorded. Peripheral skin temperature decreased at the time of URTI at all ages, though in the older infants it usually increased in parallel with rectal temperature during the latter part of the night, when pyrexia was most common.

Infants thus respond to URTI by heat conservation. In the younger infants the lower metabolic rate and the further decrease in this rate with URTI means that fever is rare, and their temperature may decrease on infection. In the older infants the increase in metabolic rate (from an already higher baseline) may result in fever. These differences may contribute to the increased vulnerability of the older infants to heat stress, particularly at the time of acute viral infections. (Arch Dis Child 1994; 70: 187-191)

Thermal stress has been shown to be associated with an increased risk of sudden infant death syndrome (SIDS) in a number of anecdotal reports and in two case-control studies. ${ }^{12}$ Although the mechanism of such an association remains unclear, the reported effects of the thermal environment on respiratory pattern suggest that, at least in some subjects, the mechanism may involve an interaction between thermoregulation and respiratory control. ${ }^{3}$ In this context the head, as the site of about $40 \%$ of heat production and up to
$85 \%$ of heat loss for the infant in bed, may be particularly vulnerable to the effects of thermal stress. ${ }^{4}$ In two studies an excess incidence of head covering was found in infants who died from SIDS compared with matched controls, ${ }^{56}$ and in animal studies relatively small changes in brain temperature have been shown to have potentially major effects on the control of respiration. ${ }^{7}$ Thus factors which increase the resting metabolic rate might have a significant influence on respiratory control both by a 'feed forward' effect of increased carbon dioxide production (which may directly affect respiratory drive) and by an effect on cerebral temperature, particularly under conditions (for example a prone sleeping position, head covering) in which the ability to lose heat from the head might be compromised. 367

The change in metabolic rate over the first three months after birth, which, when expressed in terms of heat loss for each unit of surface area amounts to an increase of over $50 \%$, might be expected to put infants aged 3 months at a higher risk of the adverse effects of a warm environment than younger infants. ${ }^{8}$ In the Avon study the risk of SIDS was increased by the presence of heavy wrapping only for those aged more than 70 days. $^{1}$

In a subsequent study we showed that, though the presence of a positive viral culture from the upper respiratory tract or gastrointestinal tract was more common in infants with SIDS than in controls matched for age and community, this did not achieve statistical significance. The combination of heavy wrapping and a positive viral vulture was, however, associated with a highly significant increased risk. $^{5}$

The response to acute viral infections in adults includes a combination of a temporary 'resetting' of the thermal set point and a number of physiological adaptations which allow the temperature to increase. These adaptations include an increase in metabolic rate and a reduction in heat loss by peripheral vasoconstriction. The relative contributions of these adaptations in newborn animals varies from one species to another. ${ }^{3}$ Little information exists on the pattern of response in the human infant, though it is known that febrile responses to acute viral infections are relatively uncommon in infants less than 3 months of age, in whom serious infection is often accompanied by a decrease in body temperature.

We report a prospective longitudinal study of the metabolic rate and thermal balance of a small group of normal infants, in whom recordings were made before, during, and after episodes of clinically identified acute infections. 


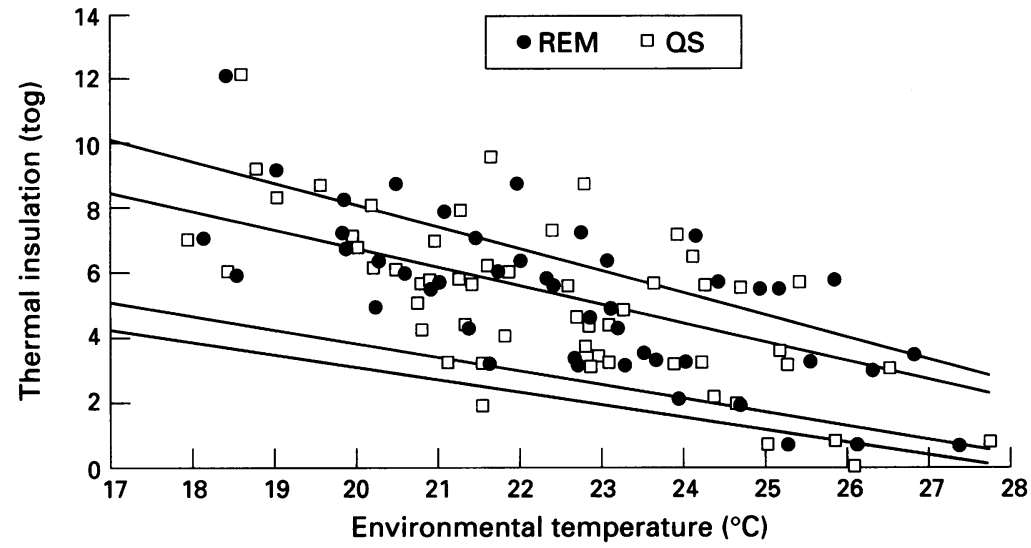

Figure 1 Comparison of the thermal insulation of the bedding and clothing added by parents at home with the values predicted from laboratory studies as representing the range of thermal neutrality. The data points represent recordings at ages between 1 week and 5 months during health. The lower two lines represent the calculated values for the lower end of the thermoneutral range for infants within the first week after birth (upper line) and at 3 months old. The upper two lines represent positions equivalent to the highest temperatures shown to be within the thermoneutral range in our laboratory studies for infants of the same ages. Values below the lower lines represent conditions below the thermoneutral range, whereas those above the lines do not necessarily represent conditions above the upper limit of thermoneutrality. ${ }^{11} Q S=$ quiet sleep; $R E M=$ rapid eye movement sleep.

\section{Subjects and methods}

A group of 17 infants, each of whom had at least one older sibling, was identified from the postnatal wards of St Michael's Hospital. All were born at term to healthy mothers with no major complications during pregnancy. Informed consent was obtained from the parents for the infant to be enrolled in the study, which was approved by the hospital research ethical committee.

Recordings were made at home, within the first week after birth, and again at the ages of 1 , 3 , and 5 months. The families were asked to call us whenever an older sibling developed signs of an upper respiratory tract infection (URTI) and arrangements were then made to make a recording of the infant within the next 24-48 hours, again after four to six days, and again two weeks later if the infant showed signs of having developed an URTI. The aim was to achieve longitudinal observations of the metabolic rate and thermal balance during health, with additional recordings before, during, and after each URTI. A detailed assessment of signs and symptoms of illness (modified from the 'Baby Check' scoring system) was carried out at each home visit. ${ }^{9} 10$ The presence of at least two of four respiratory signs/symptoms (runny nose, coughing bouts on more than five occasions, inspiratory recession, expiratory wheeze) during the

Oxygen consumption $\left(\dot{V}_{2}\right)$ and carbon dioxide production $\left(\dot{V} \mathrm{CO}_{2}\right)$ in different sleep states in the first five months after birth

\begin{tabular}{|c|c|c|c|c|c|c|}
\hline $\begin{array}{l}\text { Age } \\
\text { group }\end{array}$ & $\begin{array}{l}\text { Median } \\
\text { (range) } \\
\text { age (days) }\end{array}$ & $\begin{array}{l}\text { Sleep } \\
\text { state }^{\star}\end{array}$ & $\begin{array}{l}\text { Mean (SD) } \\
\mathrm{VO}_{2} \\
(\mathrm{ml} / \mathrm{kg})\end{array}$ & $\begin{array}{l}\text { Mean (SD) } \\
V \mathrm{CO}_{2} \\
(\mathrm{mlkg})\end{array}$ & $\begin{array}{l}\text { Mean (SD) } \\
V_{2} \mathrm{O}_{2} \\
\left(\mathrm{mV} \mathrm{m}^{2}\right)\end{array}$ & $\begin{array}{l}\text { Mean (SD) } \\
V_{C O_{2}} \\
\left(\mathrm{mV} \mathrm{m}^{2}\right)\end{array}$ \\
\hline $\bar{F}$ & 10 & $\begin{array}{l}\text { QS } \\
\text { RE } \Lambda\end{array}$ & & & & \\
\hline 1 Month & $35(27-45)$ & QS & $\begin{array}{l}7.38(0.85) \\
7.95(1.25)\end{array}$ & $\begin{array}{l}6.86 \\
7 \cdot 64\end{array}$ & $\begin{array}{l}122.3(12.0) \\
131.6(17.5)\end{array}$ & $\begin{array}{l}113.6(10.9) \\
126.6(16.5)\end{array}$ \\
\hline 3 Months & $89(85-116)$ & $\begin{array}{l}\text { QS } \\
\text { REM }\end{array}$ & $\begin{array}{l}7.51(0.67) \\
8.34(1.30)\end{array}$ & $\begin{array}{l}6.68(0.69) \\
7.72(1.18)\end{array}$ & $\begin{array}{l}138.4(13.0) \\
154.0(19.1)\end{array}$ & $\begin{array}{l}123.2(13.9) \\
142.7(17.9)\end{array}$ \\
\hline 5 Months & $155(140-201)$ & & $\begin{array}{l}7.27(0.78) \\
7.73(9.95)\end{array}$ & $\begin{array}{l}6.69(0.77) \\
7.16(1.09)\end{array}$ & $\begin{array}{l}140.8(11.7) \\
151.0(16.3)\end{array}$ & $\begin{array}{l}129.4(12.3) \\
139.6(18.4)\end{array}$ \\
\hline
\end{tabular}

${ }^{\star} \mathrm{QS}=$ quiet sleep; $\mathrm{REM}=$ rapid eye movement sleep. previous 24 hours was taken as evidence of an URTI.

Each recording consisted of an overnight recording of the infant's body temperature and the environmental temperature using a digital datalogger (Squirrel model 1202, Grant Instruments, Cambridge) as described previously. ${ }^{11}$ The temperature of the infant was determined from temperature probes sited in the rectum, axilla, shin, abdomen, and chest. Two environmental probes were sited in the air adjacent to the infant's cot, and two on the two walls closest to the cot. The output of each probe was recorded at one minute intervals. The temperature recording was continued during a daytime sleep period, starting shortly after a feed. During this sleep period a continuous recording was made of the infant's metabolic rate using an open circuit indirect calorimeter system described previously. ${ }^{12}$ This system gives values for oxygen consumption, carbon dioxide production, respiratory quotient, and calculated total energy expenditure for each minute of the recording. During the course of this recording the infant was constantly observed by the research midwife, who noted the sleep state every minute using behavioural criteria. ${ }^{13}$ The amount of clothing and bedding was determined by the infant's mother, in accordance with her usual practice. A detailed note was taken of the bedding and clothing, but no attempt was made to influence the mother's choices. After achieving a steady state within the calorimeter hood, which usually took five to 10 minutes, the first section of 10 consecutive minutes in each sleep state, quiet sleep and rapid eye movement sleep, was taken as being representative of the metabolic rate during that state. Because of the known increase in metabolic rate after feeding ${ }^{14}$ the chosen sections of quiet sleep and rapid eye movement sleep were chosen, as far as possible, to start at least 45 minutes after the feed.

\section{Results}

Of the 17 infants initially enrolled in the study, recordings were successfully completed to the age of 5 months in 12. All infants were born at term (38-41 weeks' gestation). The median

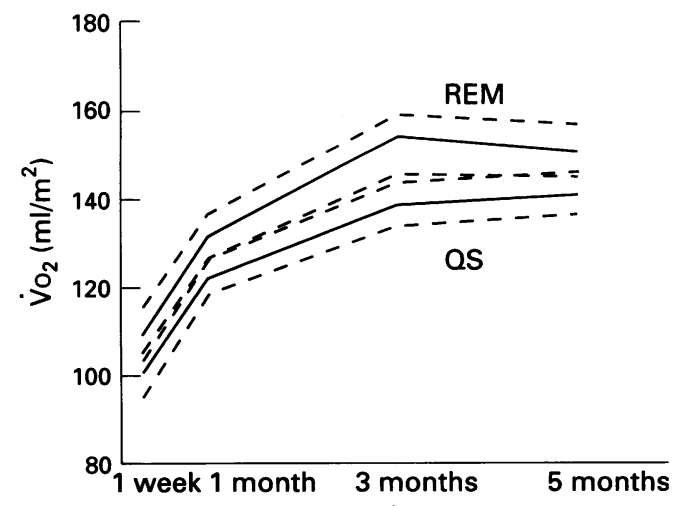

Figure 2 Oxygen consumption ( $\left.\dot{V}_{2}\right)$ during health for infants at home, in rapid eye movement sleep (REM) and quiet sleep $(Q S)$ at ages from 1 week to 5 months. The graphs show the mean ( $95 \%$ confidence interval) at each age. 

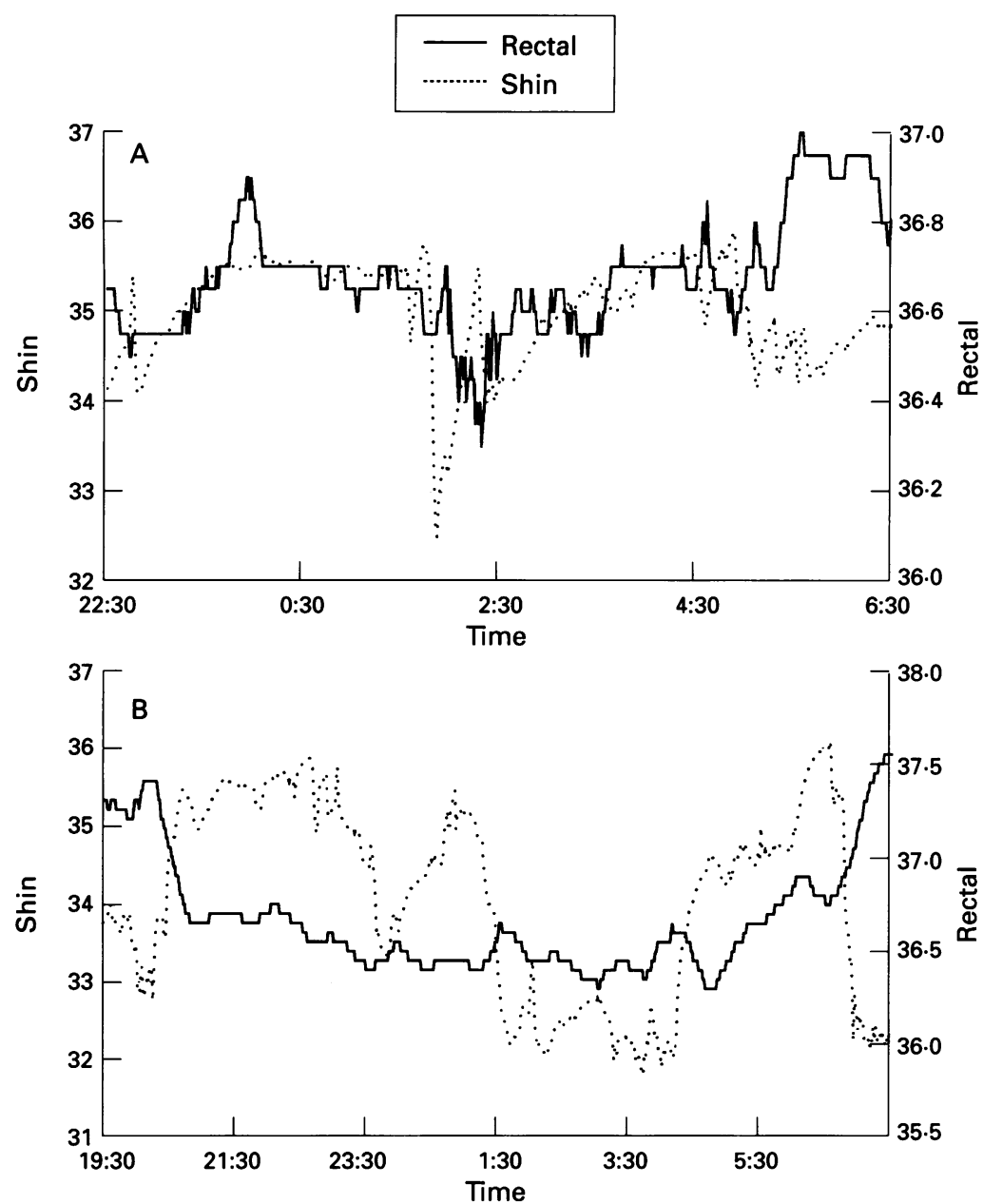

Figure 3 Overnight recordings of rectal and shin temperature in healthy infants at $(A) 30$ and $(B) 184$ days of age. peripheral skin temperature tended to increase in the early part of the night, reflecting the active cooling process which accompanied the decrease in rectal temperature, although this pattern was not seen in the younger infants. The results of these developmental changes in overnight body temperature observed in the current study and in a larger scale community based study will be presented in detail elsewhere, and will not be considered further here.

A total of 13 episodes of clinically identified URTI in the infants were included in this study. Parents usually noted a decreased feed intake at the time of an apparent URTI. Six of these episodes occurred in infants aged less than 3 months, and seven episodes in infants aged 3 to 6 months. Three infants had two episodes of URTI for each of which recordings of metabolic rate were obtained before, during, and after the episode. None of the infants aged less than 3 months became febrile (rectal temperature $>38^{\circ} \mathrm{C}$ ) at the time of an URTI, although three of the older infants became febrile. Figure 4 shows typical overnight recordings of rectal and shin temperature for the same infants as in fig 3, at the times of infections at 64 and 148 days. In both age groups the skin temperature was lower in the early part of the night at the time of the infection, indicating peripheral vasoconstriction and heat conservation. In the older infants the decrease in rectal temperature was less at the time of the infection than when well, and during the latter part of the night the rectal temperature usually increased to more than $37.5^{\circ} \mathrm{C}$.

Assessment of the effect of URTI on metabolic rate was complicated by the relatively wide differences between infants, between sleep states in the same infant, and by the changes with age. Comparisons were therefore only made between the values obtained for metabolic rate on different occasions in the same infant in the same sleep state. The change in oxygen consumption attributable to the presence of an URTI was assessed by calculating the difference between the value observed during the URTI and the expected value for the same child at that age, as deduced by interpolation from recordings made before and after the infection.

When expressed in this way there were marked age dependent differences in the effect of infection on metabolic rate. In five of the six infants aged less than 3 months the metabolic rate showed no significant change or decreased at the time of URTI, though in three of the five infants older than 4 months there was a significant increase in metabolic rate, the other two infants showing no significant change. Figure 5 shows these changes for the 11 episodes for which satisfactory recordings were obtained in quiet sleep before, during, and after the URTI. There is a significant increase with age in the tendency for metabolic rate to increase with URTI ( $<<0.005$; Spearman rank order correlation).

\section{Discussion}

The upper limit of thermal neutrality could not be reliably defined from our laboratory 

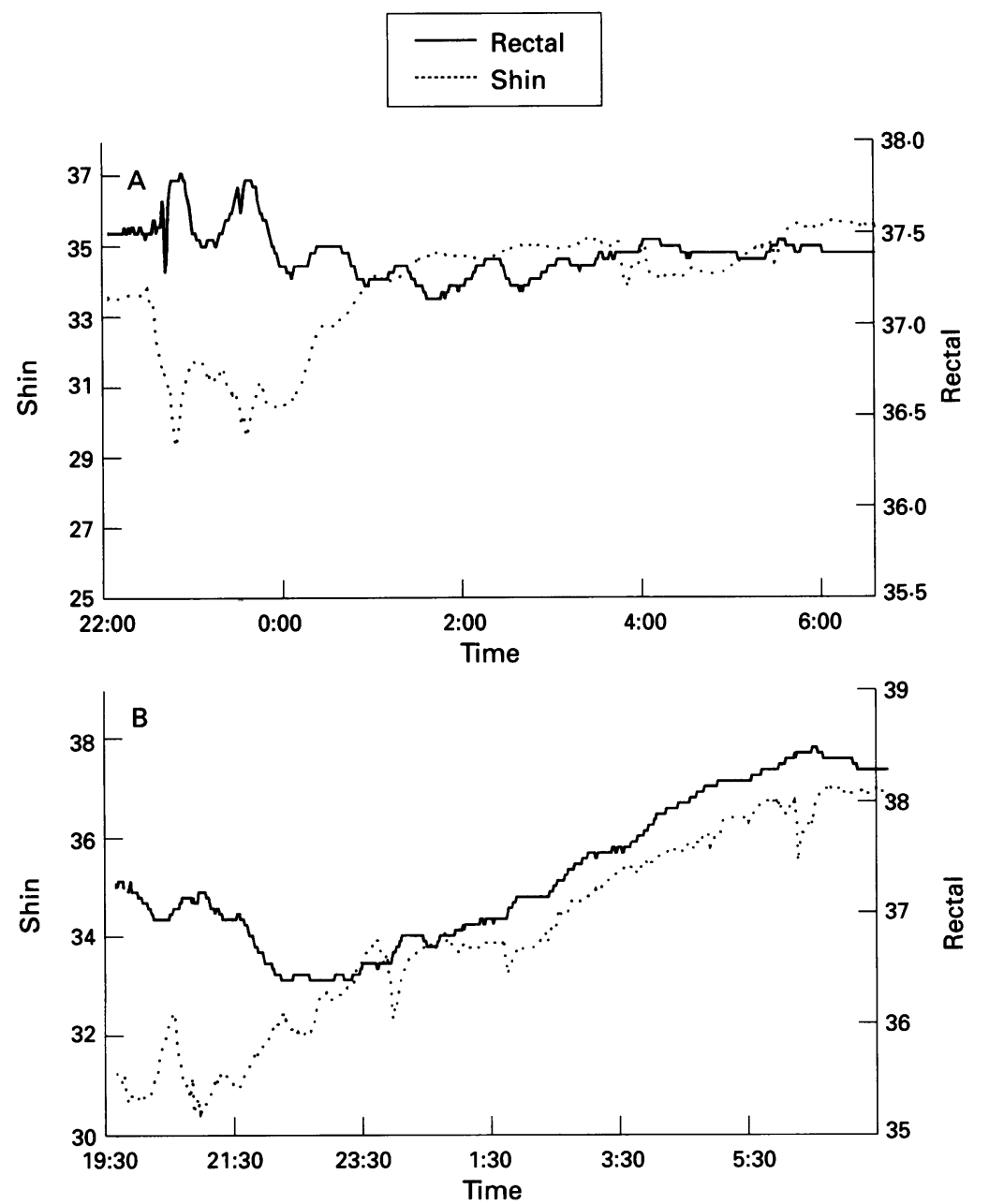

Figure 4 Overnight recordings of rectal and shin temperature in the same infants as in fig 3 at the time of URTI at $(A) 64$ and $(B) 148$ days of age.

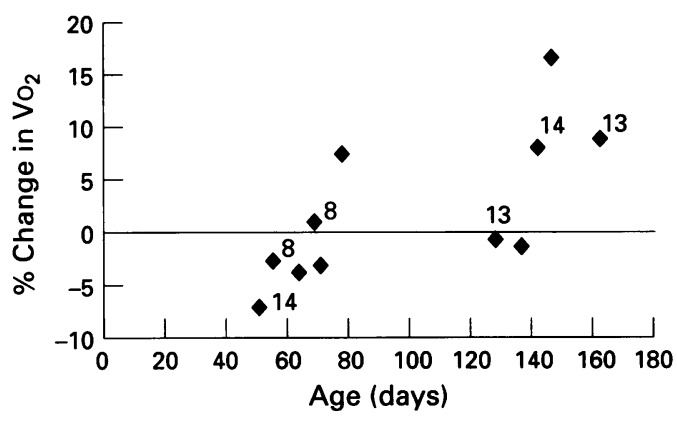

Figure 5 Percentage change in oxygen consumption $\left(\mathrm{VO}_{2}\right)$ at the time of URTI in quiet sleep for the 11 episodes for which satisfactory recordings were obtained before, during, and after the episodes. There is an increase with age in the tendency for $\dot{V} O$ to increase with URTI $(p<0.005$

Spearman rank order correlation). The numbers represent the recordings from the infants (Nos $8,13,14$ ) from whom recordings were obtained in more than one URTI.

studies, ${ }^{811}$ and there was considerable interindividual variation in the lower limit. The results of this study confirm our previous observation that most mothers of young infants adjust the amount of bedding and clothing which they apply to their infants to ensure that the thermal environment is within or above the range predicted to be thermoneutral.

The sequential measurements of metabolic rate obtained from the infants in this study are in close agreement with those obtained in our previous laboratory studies (using a different methodology). This confirms the reproduci- bility of these values, and provides direct evidence that the present recordings were made under conditions of thermal neutrality. ${ }^{8}$ These data thus provide experimental validation of the theoretical model of heat balance in infants which we have previously described, and the calculated values of added bedding necessary to achieve thermoneutrality, which are derived from that model. ${ }^{11}$

A number of interesting observations emerge from the recordings obtained at the time of URTI. Firstly, we were able to confirm the well recognised clinical observation that febrile responses to minor viral infections are less common in infants in the first three months after birth than in older infants. The two age groups of infants showed evidence of increased heat conservation by peripheral vasoconstriction at the time of URTI, but only in the older infants was this accompanied by an increase in rectal temperature. This difference is likely to be related to the observed changes in metabolic rate, which decreased in the younger infants and increase in the older infants at the time of URTI. The increase in metabolic rate with infection in the older infants probably reflects the acute phase response to a viral infection, whereas the lack of such an increase in metabolic rate in some of the younger infants may reflect an immaturity of this response. The decrease in metabolic rate in some of the younger infants may represent the effect of a decreased feed intake at the time of infection.

Although the lack of virological investigations in this study limits the interpretation of the findings with respect to specific viruses, the diagnosis of acute viral URTI is almost always clinical, made on signs and symptoms of illness. In clinical practice negative viral cultures in the presence of clear clinical signs of a viral URTI would not preclude this diagnosis. As a common source of viral URTI in young infants seems to be an older sibling, the use of older siblings as an 'advance warning' of an impending URTI in the infant allowed us to obtain recordings immediately before and during such infections in this study. The rapidity of changes in metabolic rate with age in the first three months after birth meant that it was important to obtain a 'control' recording as soon before the URTI as possible. From our previous experience of trying to obtain recordings at the time of infections in infants we found that parents were reluctant to contact us when the infant was unwell. This study has shown that parents are more willing to make contact at the time of a sibling's illness, and then to allow recordings from the infant a few days later.

If a positive thermal balance and its effects on homoeostatic control mechanisms is an important factor contributing to SIDS, then the results of this study suggest that the infants most at risk are those over the age of 3 months. In our community based studies of risk factors for SIDS $^{5}$ we found that the combination of heavy wrapping and viral infection significantly increased the risk of SIDS only for those infants older than 10 weeks. The differences in 
the patterns of response to URTI between younger and older infants shown in this study are entirely consistent with this finding, though the nature of the mechanism responsible for the differences with age remain unclear.

These results emphasise the potential effects on thermal balance of heavy wrapping at the time of acute viral infections, particularly for infants older than 3 months. For younger infants the implications are less clear and further work is needed on the nature of the metabolic response to infection in the first three months after birth.

These studies were generously supported by the Foundation for the Study of Infant Deaths (project 121). We also acknowledge the Study of Infant Deaths (project 121). We also acknowledge
the assistance of Dr Peter Rudd and Professor Jean Golding in the design of the study.

1 Fleming PJ, Gilbert RE, Azaz Y, et al. The interaction between bedding and sleeping position in sudden infant death syndrome: a population-based case-control study. $B M F$ 1990; 301: 85-9.

2 Ponsonby AL, Dwyer T, Gibbons LE, et al. Thermal environment and sudden infant death syndrome: case-control study. BMF 1992; 304: 277-82.

3 Fleming PJ, Levine MR, Azaz Y, et al. Interactions between thermoregulation and the control of respiration in infants: possible relationship to sudden infant death. Acta Paediatr 1993; Suppl 389: 57-9.

4 Wailoo MP, Petersen SA, Whittaker H, Goodenough P The thermal environment in which 3-4 month old infants sleep at home. Arch Dis Child 1989; 64: 600-4.

5 Gilbert RE, Rudd PT, Berry PJ, et al. Combined effect of infection and heavy wrapping on the risk of sudden infant death. Arch Dis Child 1992; 67: 272-7.

6 Bacon CJ. Thermal environment and sudden infant death syndrome. Report of the Chief Medical Officer's Exper Group on the sleeping position of infants and cot death. London: HMSO, 1993: 68-76.

7 Hanson M, Calder N, Watanabe T, Kumar P. The drive to breath and sudden infant death syndrome. Acta Paediatr 1993; Suppl 389: 47-9.

8 Azaz Y, Fleming PJ, Levine $M$, et al. The relationship between environmental temperature, metabolic rate, sleep state and evaporative water loss in infants from birth to three months. Pediatr Res 1992; 32: 417-23.

9 Morley CJ, Thornton AJ, Cole TJ, et al. Baby check: a scoring system to grade the severity of acute systemic illness in babies under 6 months old. Arch Dis Child 1991; 66: $100-5$.

10 Cole TJ, Gilbert RE, Fleming PJ, et al. Baby check and the Avon infant mortality study. Arch Dis Child 1991; 66: 1077-8.

11 Wigfield RE, Fleming PJ, Azaz Y, et al. How much wrapping do babies need at night? Arch Dis Child 1993; 69: ping do

12 Shortland GJ, Fleming PJ, Walter JH. Validation of a portable indirect calorimetry system for measurement of energy expenditure in sick preterm infants. Arch Dis Child 1992; 67: 1207-11.

13 Prechtl HFR. The behavioural states of the newborn infant. Brain Res 1974; 76: 1304-11.

14 Stothers JK, Warner RM. Oxygen consumption and neonatal sleep state. F Physiol 1978; 278: 435-40. 